\title{
Detection and preliminary screening of the human gene expression profile for Hirschsprung's disease
}

\author{
XIN WANG ${ }^{1,2}$, SHIQI WANG ${ }^{1,2}$, XIANQING JIN ${ }^{1,2}$, NING WANG ${ }^{1,2}$, YUANYUAN LUO ${ }^{1,2}$ and YINPING TENG ${ }^{1,2}$ \\ ${ }^{1}$ Tumour Laboratory of Children's Hospital of Chongqing Medical University; \\ ${ }^{2}$ Department of Neonatal Gastrointestinal Surgery, Children's Hospital of Chongqing Medical University, \\ Chongqing 400014, P.R. China
}

Received December 5, 2014; Accepted September 1, 2015

DOI: $10.3892 / \mathrm{mmr} .2015 .4633$

\begin{abstract}
The present study investigated a genome microarray of colorectal lesions (spasm segments) in children with Hirschsprung's disease (HSCR), and analyzed the results. In addition, the present study screened for differentially expressed genes in children with HSCR. Microarray technology was used to examine the human gene expression profiles of the colorectal lesions (spasm segments) of six children with HSCR, and three normal colon tissue samples. The data were analyzed be determining P-values of significance and absolute fold changes. Preliminary screening was performed to identify genes exhibiting significant differential expression in children with HSCR,
\end{abstract}

Correspondence to: Dr Xianqing Jin, Tumour Laboratory of Children's Hospital of Chongqing Medical University, 136 Zhongshan Second Road, Chongqing 400014, P.R. China

E-mail: etzhl@163.com

Abbreviations: HSCR, Hirschsprung's disease; BP, biological process; CC, cellular component; MF, molecular function; CXCR7, chemokine (C-X-C motif) receptor 7; ATF3, activating transcription factor 3; JUN, jun proto-oncogene; DCN, decorin; HOXD13, homeobox D13; FGL2, fibrinogen-like 2; NTN4, netrin 4; UBC, ubiquitin C; ATF1, activating transcription factor 1; WNT5A, wingless-type mouse mammary tumor virus integration site family, member 5A; RGS5, regulator of G-protein signaling 5; GLI1H, GLI family zinc finger 1; VIM, vimentin; BMP3, bone morphogenetic protein 3; ZDHHC8, zinc finger, DHHC-type containing 8; ZDHHC3, zinc finger, DHHC-type containing 3; WHSC1, Wolf-Hirschhorn syndrome candidate 1; DDC, dopa decarboxylase (aromatic L-amino acid decarboxylase); GAP43, growth associated protein 43; PIK3CD, phosphoinositide-3-kinase, catalytic, delta polypeptide; SCG2, secretogranin II; RTN1, reticulon 1; ADRA2B, adrenergic, alpha-2B-receptor; PKDCC, protein kinase domain containing, cytoplasmic homolog (mouse); COBL, cordon-bleu homolog (mouse); TLR5, toll-like receptor 5; NLRP11, NOD-like receptor family, pyrin domain containing 11; TAF8, TAF8 RNA polymerase II, TATA box binding protein-associated factor; TAB3, TGF- $\beta$ activated kinase 1/MAP3K7 binding protein 3

Key words: Hirschsprung's disease, gene chip, genome microarray, screening and these target genes were analyzed in subsequent verification and analytical investigations. Of $>20,000$ detected human genes, the preliminary screenings demonstrated that 3,850 genes were differentially expressed and upregulated, with $\mathrm{P}<0.05$ and $>2$-fold absolute changes in expression. In addition, 645 differentially expressed genes with $\mathrm{P}<0.05$ and $>2$-fold absolute changes were downregulated. Of the upregulated genes, 118 were involved in classic signaling pathways, compared with 11 of the downregulated genes $(\mathrm{P}<0.001$; absolute fold change $>2$-fold). HSCR etiology is complex and often involves multiple gene changes. Microarray technology can produce large quantities of gene expression data simultaneously, and analyzing this data using various techniques may provide a fast and efficient method for identifying novel gene targets and for investigating the mechanisms underlying HSCR pathogenesis.

\section{Introduction}

Congenital intestinal aganglionosis, which is also referred to as Hirschsprung's disease (HSCR) is a common gastrointestinal deformity in children that is caused by defects that occur during the migration, proliferation, differentiation, survival and apoptosis of gut neural crest stem cells (1). HSCR has an incidence of 1/5,000 live births and a male:female ratio of $\sim$ 4:1 (2). Epidemiological and animal studies have demonstrated that the etiology of the disease is associated with various genes, including the proto-oncogene, RET, endothelin signaling gene and transcription factors $(3,4)$. However, the exact mechanism underlying the pathogenesis of HSCR remains to be fully elucidated. In the present study, human cDNA microarray technology was applied, and the results were comparatively analyzed using various techniques to determine differences in gene expression profiles on the same chip between normal colon tissue samples and those from patients with HSCR. This involved the preliminary screening for genes with differential expression levels in the colorectal lesions of children with HSCR, which were compared with normal colon tissue samples. Differences in gene expression may provide information regarding HSCR candidate genes.

\section{Materials and methods}

Specimen collection. The subjects of the present study included six children with HSCR (five males and one 
female, aged between 13 days and 4 years old) with no other concurrent abnormalities. The children were recruited from the Department of Neonatal Gastrointestinal Surgery at the Children's Hospital of Chongqing Medical University (Chongqing, China). Written-informed consent was obtained from the family of the patients. Under sterile surgical conditions, full-thickness bowel tissues were cut from the stricture, expansion and anastomotic sections, and trimmed into tissue blocks $(0.3-0.5 \times 0.5-1.0 \mathrm{~cm})$. These tissue samples were placed in sterile aluminum foil for rapid storage in liquid nitrogen. The stricture was located and used as the experimental tissue specimen. The control group included three randomly selected male cases (from the original five males). The normal colon tissue samples were used as control specimens, and were collected from colorectal trauma cases and from the stoma during leakage closure following congenital anorectal transverse colostomy. The control samples were sectioned in a similar manner to the patient samples. The study was approved by the ethics committee of the Children's Hospital of Chongqing Medical University.

Array information. An Agilent Human 4x44 K Gene Expression Microarray v.2 was supplied by KangChen Bio-tech Inc. (Shangha, China), and used to perform the gene expression profiles of the nine human tissue samples (project code $\mathrm{H} 1305022$ ).

The whole human genome oligo microarray represents all known genes and transcripts of the human genome. The sequences were compiled from a broad source survey prior to verification and optimization via alignment to the assembled human genome. Coupled with Agilent probe selection and a robust validation processes, this method provides increased data quality and reduces gene-coverage redundancy (unpublished data). The targets were 27,958 Entrez Gene RNAs. The content was sourced from RefSeq Build 36.3 (http:// www.ncbi.nlm.nih.gov/refseq/), Ensemble Release 52 (http:// asia.ensembl.org/index.html?redirect=no), Unigene Build 216 (http:/www.ncbi.nlm.nih.gov/unigene/) and GenBank (http:// www.ncbi.nlm.nih.gov/genbank).

RNA extraction and quality control. The tissue samples were cryopulverized using BioPulverizer ${ }^{\mathrm{TM}}$ (BioSpec Products Inc, Bartlesville, OK, USA) prior to being placed in liquid nitrogen. The tissue samples were then further homogenized using a Mini-Bead-Beater-16 (BioSpec Products Inc). Total RNA was extracted using TRIzol ${ }^{\circledR}$ (Invitrogen; Thermo Fisher Scientific, Inc., Waltham, MA, USA) and an RNeasy Mini Spin column (Qiagen, Shanghai, China) according to the manufacturer's protocol. RNA integrity and concentration were assessed following RNA extraction and prior to sample labeling. RNA integrity was assessed using agarose gel electrophoresis (Enzo Life Sciences, Inc., Farmingdale, NY, USA). Intact total RNA separated on a denaturing gel exhibits distinct $28 \mathrm{~S}$ and $18 \mathrm{~S}$ rRNA bands (eukaryotic samples), with the 28S rRNA band being approximately twice as intense as the $18 \mathrm{~S}$ rRNA band. This 2:1 intensity ratio indicates that the RNA is intact (unpublished data). A NanoDrop ND-1000 spectrophotometer was used to accurately measure the RNA concentrations at optical density (OD)260, protein contamination (ratio of OD260/OD280) and organic compound contamination (ratio of OD260/OD230). The results typically provided total RNA with an OD260/OD280 ratio $>1.8$.

The whole procedure was performed in accordance with manufacturer's instruction, including RNA sample preparation, total RNA clean-up and quality control, labeling reaction preparation, purification of the labeled/amplified RNA, labeled cRNA quality control, hybridization, microarray washing and scanning. The specific activity ( $\mathrm{pmol}$ dyes $/ \mu \mathrm{g}$ genomic DNA) of the labeled genomic DNA was obtained using the following equation: Specific activity $=($ concentration of $\mathrm{Cy} 3) /$ (concentration of cRNA) $x 1,000=$ pmol $\mathrm{Cy} 3$ per $\mu \mathrm{g}$ cRNA. If the yield was $<1.65 \mu \mathrm{g}$, and the specific activity was $<9.0 \mathrm{pmol}$ $\mathrm{Cy} 3 / \mu \mathrm{g}$ cRNA, the hybridization step did not proceed, and re-preparation of the cRNA was required.

RNA labeling and in situ hybridization. Sample labeling and array hybridization were performed using Agilent One-Color Microarray-Based Gene Expression Analysis (Agilent Technologies, Inc., Santa Clara, CA, USA), according the manufacturer's instructions. The total RNA from each sample was linearly amplified and labeled using Cy3-UTP (Enzo Life Sciences). The labeled cRNAs were purified using an RNeasy Mini kit (Qiagen). The concentration and specific activity levels of the labeled cRNAs (pmol Cy3/ $\mu \mathrm{g}$ cRNA) were measured using the NanoDrop ND-1000 spectrophotometer. A total of $1 \mu \mathrm{g}$ of each labeled cRNA was fragmented by adding $11 \mu 1$ 10X blocking agent (Agilent Technologies, Inc.) and $2.2 \mu \mathrm{l}$ of $25 \mathrm{X}$ fragmentation buffer (Agilent Technologies, Inc.). The mixture was then heated to $60^{\circ} \mathrm{C}$ for $30 \mathrm{~min}$, and $55 \mu 12 \mathrm{X}$ GE hybridization buffer (Agilent Technologies, Inc.) was added to dilute the labeled cRNA. A total of $100 \mu \mathrm{l}$ hybridization solution was dispensed into a gasket slide, which was added to the gene expression microarray slide. The slides were incubated for $17 \mathrm{~h}$ at $65^{\circ} \mathrm{C}$ in an Agilent hybridization oven (Agilent Technologies, Inc.). The hybridized arrays were washed with Milli-Q water (Millipore, Billerica, MA, USA), fixed, and scanned using an Agilent DNA Microarray Scanner (G2505C; Agilent Technologies, Inc.).

An Agilent Quick Amp Labeling kit (Agilent Technologies, Inc.) was used for the sample labeling. Hybridization was performed in Agilent SureHyb Hybridization Chambers (Agilent Technologies, Inc.). In GeneSpring GX v11.5.1, a normalized value is a relative number that comes from the ratio of the comparison between the raw value of the listed probe and that of the controls. Normalized values were calculated based on the normalization method applied to the data. Please refer to the technical note for information on how the control values were calculated. For one-color data, the four normalization methods (median, scale, quantile, and normalization to control genes) were used. For two-color data, each raw intensity value corresponding to the control channel was adjusted using a locally-weighted Lowess regression method. Each value in the signal channel was divided by the adjusted control value, resulting in the final normalized value.

Data collection and analysis. The slides were washed with Milli-Q water and subsequently scanned using the Agilent DNA Microarray Scanner (Agilent Technologies, Inc.). Data was collected using Agilent Feature Extraction software (Agilent Technologies, Inc.). Normalization and data analysis 
Table I. RNA quantification and quality assurance.

\begin{tabular}{|c|c|c|c|c|c|c|}
\hline Sample ID & OD260/280 ratio & OD260/230 ratio & Conc. $(\mathrm{ng} / \mu \mathrm{l})$ & Volume $(\mu 1)$ & Quantity (ng) & QC pass or fail \\
\hline 2 & 2.01 & 2.29 & $1,002.25$ & 80 & $80,180.00$ & Pass \\
\hline 3 & 1.99 & 1.98 & 534.28 & 10 & $5,342.80$ & Pass \\
\hline 4 & 1.99 & 2.29 & $1,084.86$ & 80 & $86,788.80$ & Pass \\
\hline 10 & 1.99 & 2.32 & 954.17 & 60 & $57,250.20$ & Pass \\
\hline 13 & 1.98 & 2.24 & 653.52 & 50 & $32,676.00$ & Pass \\
\hline 16 & 1.99 & 2.26 & 914.64 & 80 & $73,171.20$ & Pass \\
\hline 20 & 1.93 & 2.25 & 593.50 & 10 & $73,171.20$ & Pass \\
\hline 21 & 1.91 & 2.28 & 538.83 & 20 & $10,776.60$ & Pass \\
\hline 26 & 2.00 & 2.29 & $1,237.42$ & 60 & $74,245.20$ & Pass \\
\hline
\end{tabular}

For spectrophotometery, pure RNA has an OD ratio of A260/A280 of 2.0 (ratios between 1.8 and 2.1 are acceptable). The required OD ratio of A260/A230 is $>1.8$. OD, optical density; QC, quality control.

Table II. Labeling efficiency quality control.

\begin{tabular}{|c|c|c|c|c|c|c|}
\hline Sample ID & Dye name & Dye $(\mathrm{pmol} / \mu \mathrm{l})$ & $\begin{array}{c}\text { cRNA } \\
\text { concentration }(\mu \mathrm{g} / \mu \mathrm{l})\end{array}$ & $\begin{array}{l}\text { Specific activity }^{\mathrm{a}} \\
\text { (pmol dye/ } \mu \mathrm{g} \text { cRNA) }\end{array}$ & Volume $(\mu 1)$ & $\begin{array}{c}\text { Total } \\
\text { quantity }(\mu \mathrm{g})\end{array}$ \\
\hline 2 & $\mathrm{Cy} 3$ & 16.69 & 0.74462 & 22.41412 & 20 & 14.892 \\
\hline 3 & Сy3 & 16.79 & 0.72459 & 23.17172 & 20 & 14.492 \\
\hline 4 & Сy3 & 16.71 & 0.76486 & 21.84714 & 20 & 15.297 \\
\hline 10 & $\mathrm{Cy} 3$ & 16.59 & 0.72675 & 22.82766 & 20 & 14.535 \\
\hline 13 & Cy3 & 16.13 & 0.71639 & 22.51567 & 20 & 14.328 \\
\hline 16 & $\mathrm{Cy} 3$ & 16.85 & 0.76996 & 21.88425 & 20 & 15.399 \\
\hline 20 & Cy3 & 16.77 & 0.74127 & 22.62334 & 20 & 14.825 \\
\hline 21 & Cy3 & 16.36 & 0.79604 & 20.55173 & 20 & 15.921 \\
\hline 26 & Cy3 & 16.31 & 0.70736 & 23.05757 & 20 & 14.147 \\
\hline
\end{tabular}

${ }^{a}$ For two-color samples, if yield $<825 \mathrm{ng}$ and specific activity $<8.0 \mathrm{pmol} \mathrm{Cy} 3$ or Cy5$/ \mu \mathrm{g}$ cRNA, hybridization did not proceed and the cRNA was re-prepared. For one-color samples, if yield $<1.65 \mu \mathrm{g}$ and specific activity $<9.0 \mathrm{pmol} \mathrm{Cy} 3$ or Cy $5 / \mu \mathrm{g}$ cRNA, hybridization did not proceed and the cRNA was re-prepared.

were performed using Agilent GeneSpring GX v11.5.1 (Agilent Technologies, Inc.). The results were provided in the Gene Expression Profiling Data.xls file and Data Analysis Folder. Agilent Feature Extraction software v11.0.1.1 (Agilent Technologies, Inc.) was used to analyze the acquired array images. Quantile normalization and subsequent data processing were performed using the GeneSpring GX v11.5.1 software package (Agilent Technologies, Inc.). Following quantile normalization of the raw data, genes for which a minimum of three out of the nine samples were statistically significant were selected for further data analysis. Genes that were differentially expressed in the two groups to a statistically significant degree were identified using volcano plot filtering. Hierarchical clustering was performed using Agilent GeneSpring GX software v11.5.1 (Agilent Technologies, Inc.). Gene ontology (GO) analysis and pathway analysis were performed using a standard enrichment computation method. The results were analyzed for three parameters: Molecular function, biological process and cellular components. The analyses were based on volcano plots, box plots, scatter plots,

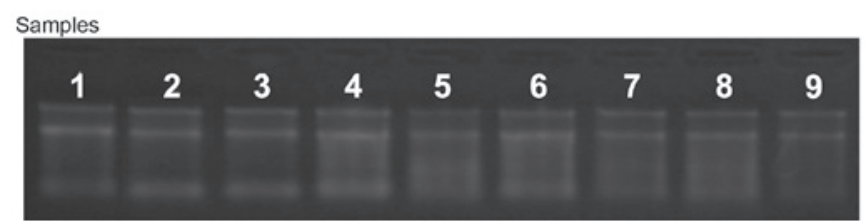

Figure 1. RNA integrity and gDNA contamination assessment using denaturing agarose gel electrophoresis. Lanes 1-9 represent total RNA of samples $2,3,4,10,13,16,20,21$, and 26 , respectively.

heat maps, hierarchical clustering, P-values, absolute fold change and GO assessed. Preliminary screening was used to determine the genes with statistically significant differences in expression levels in children with HSCR, in order to determine target genes for further verification and analysis step.

\section{Results}

Total RNA extraction and quality assurance. A NanoDrop ND-1000 spectrophotometer was used to accurately measure 


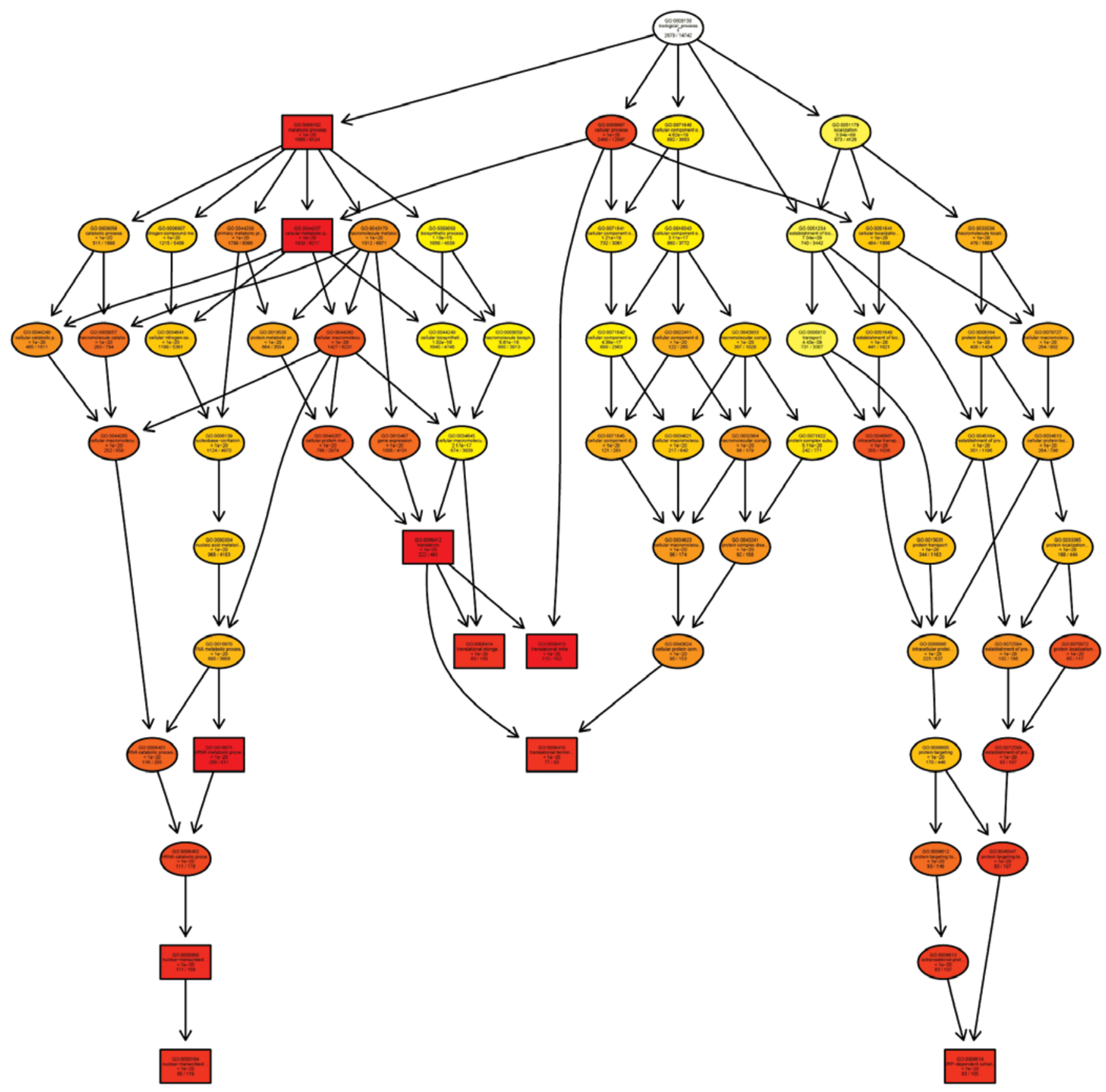

Figure 2. GO analysis of the upregulated biological process category in the HSCR investigation. The GO Directed Acyclic Graph graphical output shows the enriched GO terms in the upregulated biological process category for the gene network in the HSCR investigation. The boxes represent GO terms; with the GO ID on the top line, definition and information on the second line and P-value on the third line. The fourth line is labeled with the gene count associated with the listed GO ID (directly or indirectly) on the selected platform and the total number of genes in the dataset. Annotation moves from more general to more specific with the progression from parent nodes. A gene annotated to a specific node was also considered to be annotated at the parent nodes. The top 10 terms with the lowest P-values and their parent nodes are shown; with the terms with pane marks being significantly enriched. The degree of color saturation for each node is positively correlated with the enrichment significance of the corresponding GO term. Round nodes represent a biological process. The more red-saturated the node is, the greater its significance. <1e-20, P-value <1e-20; GO, gene ontology; HSCR, Hirschsprung's disease.

the RNA concentrations (OD260), protein contamination (ratio of OD260/OD280) and organic compound contamination (ratio of OD260/OD230). The results typically provided total RNA with an OD260/OD280 ratio $>1.8$. RNA integrity was assessed using agarose gel electrophoresis. Intact total RNA separated on the denaturing gel exhibited marked 28S and 18S rRNA bands (eukaryotic samples), with the 28S rRNA being approximately twice as intense as the 18S rRNA band. This 2:1 intensity ratio indicated that the RNA was intact (Tables I and II; Fig. 1). A total of $1 \mu \mathrm{g}$ RNA was used for labeling and the specific activity levels (pmol dye/ $\mu \mathrm{g}$ cRNA) of the labeled RNA was determined.

Determination of normalized values from the initially loaded raw data. In GeneSpring GX v11.5.1, a normalized value is a relative number, originating from the raw value ratio of the listed probe of the control (unpublished data). 


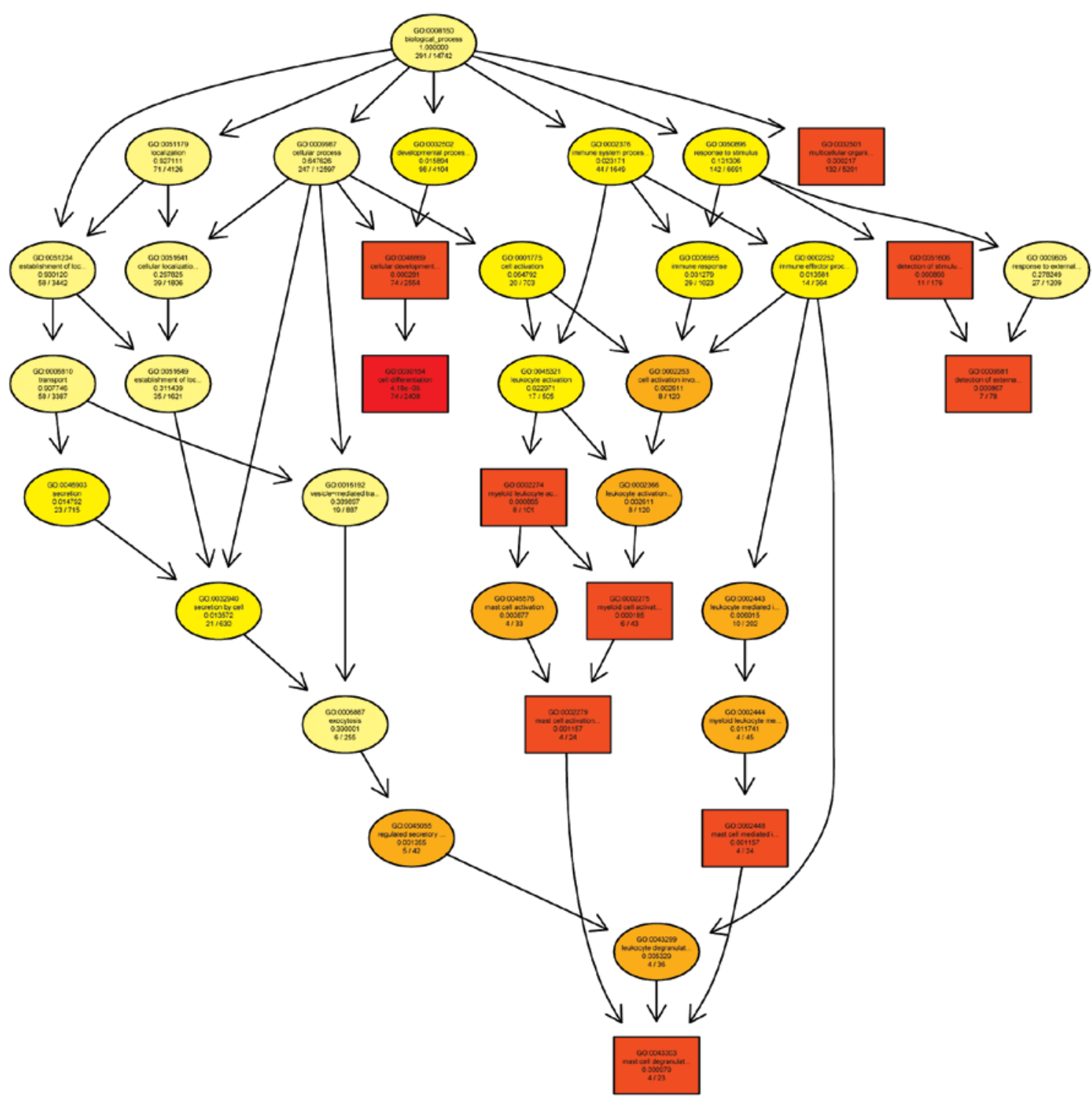

Figure 3. GO analysis of the downregulated biological process category of the HSCR investigation. The GO Directed Acyclic Graph graphical output shows the enriched GO terms in the downregulated biological process category for the gene network selected in the present HSCR investigation. The boxes represent GO terms and are labeled with the GO ID on the first line, the term definition and information on the second line and the P-value on the third line. The fourth line is labeled with the number of genes associated with the listed GO ID (directly or indirectly) on the selected platform and the total number of genes in the dataset. Annotation moves from more general to more specific with progression from the parent nodes. A gene annotated to a specific node was also considered to be annotated at the parent nodes. The top 10 terms with the lowest P-values and their parents are shown; with the terms with pane marks being significantly enriched. The degree of color saturation for each node is positively correlated with the enrichment significance of the corresponding GO term. The more red-saturated a node is, the greater its significance. in the GO DAG. <le-20, P-value <1e-20; GO, gene ontology; HSCR, Hirschsprung's disease.

Genes with differential expression levels. Microarray technology was used to examine the human gene expression profiles of the colorectal lesions (spasm segment) of children with HSCR, compared with the normal colon tissue samples, used as a control. In the present study, the primary aim was to determine the biological processes underlying the pathology of the disease, particularly regarding growth-associated genes and their association with signaling pathways (Figs. 2 and 3).

Of $>20,000$ detected human genes, preliminary screening revealed 3,850 differentially expressed genes with upregulated expression levels, $(\mathrm{P}<0.05$; absolute fold changes $>2$-fold $)$. Among those upregulated genes, the P-values were as follows: $\mathrm{P}=0.01-0.05$ for 154 genes, $\mathrm{P}=0.001-0.009$ for 468 genes, $\mathrm{P}=0.0001-0.0009$ for 834 genes and $\mathrm{P}<0.0001$ for 2,394 genes.
The absolute fold changes were $>10$ for 28 genes, 5-10 for 286 genes, 3-5 for 1,343 genes, and $<3$ for 2,193 genes.

A total of 645 differentially expressed genes with downregulated expression levels were identified, $(\mathrm{P}<0.05$; absolute fold changes $>2$-fold). Among the downregulated genes, the P-values were as follows: $\mathrm{P}=0.01-0.05$ for 52 genes, $\mathrm{P}=0.001-0.009$ for 111 genes, $\mathrm{P}=0.0001-0.0009$ for 142 genes and $\mathrm{P}<0.0001$ for 321 genes. The absolute fold changes were $>10$ for 13 genes, 5-10 for 37 genes, 3-5 for 93 genes, and $<3$ for 483 genes.

Of the upregulated genes, 118 were involved in widely-recognized signal transduction pathways $(\mathrm{P}<0.01)$, including 48 genes with $\mathrm{P}=0.01-0.0001$ and absolute fold changes $>2$-fold and 70 genes with $\mathrm{P}<0.0001$ and absolute fold changes $>2$-fold. Of the downregulated genes, 11 genes 


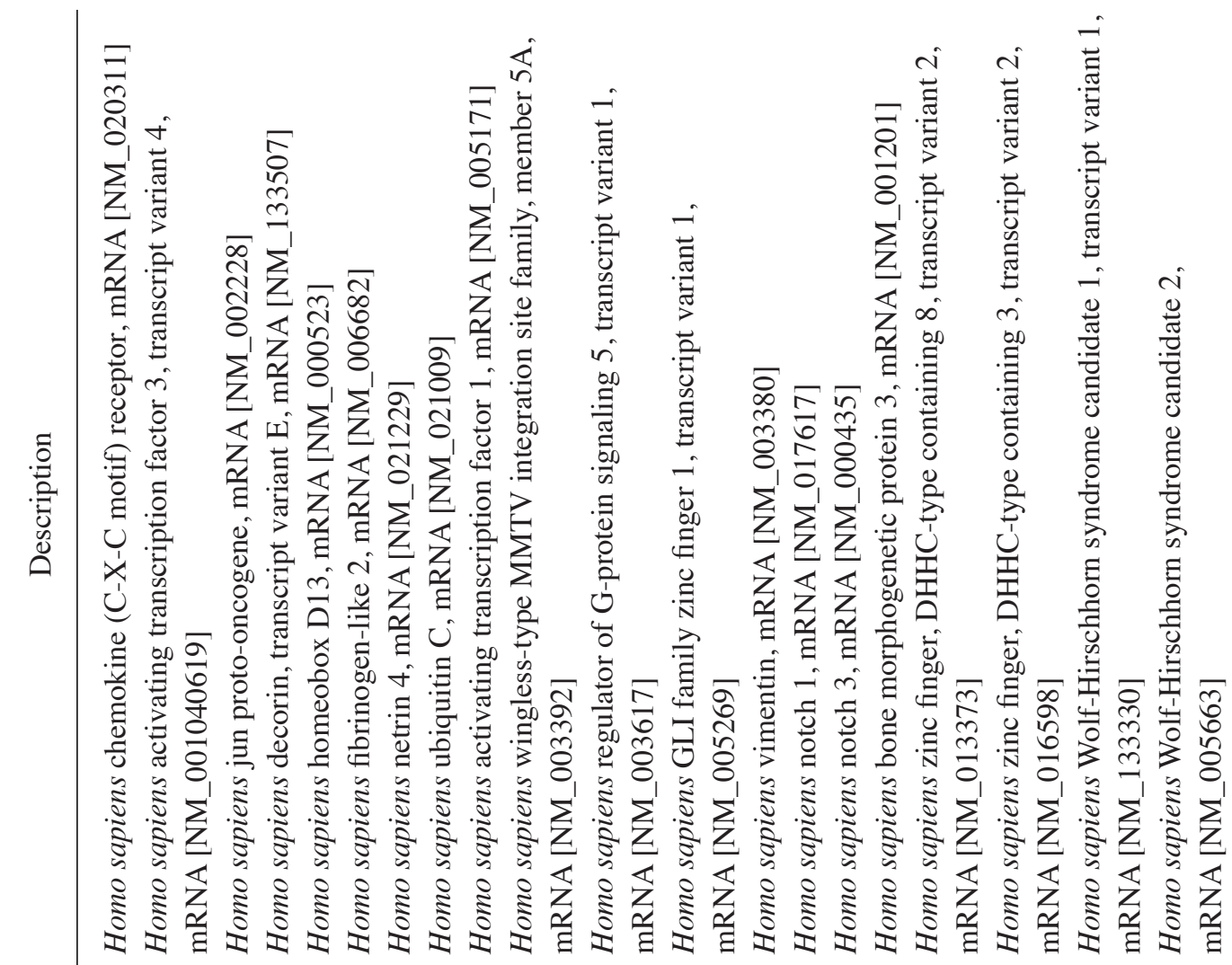

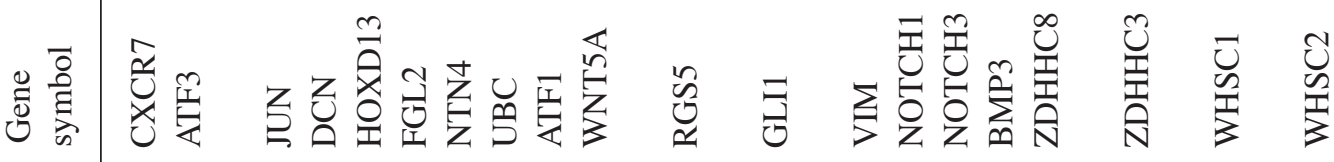

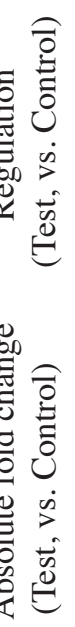

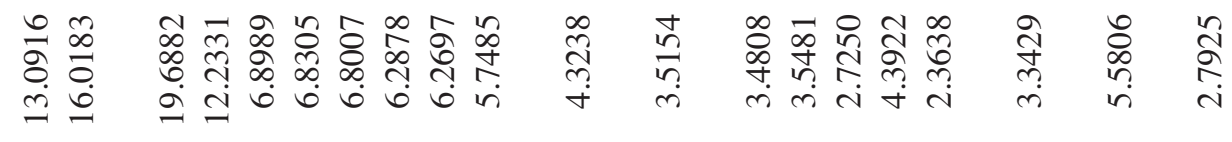

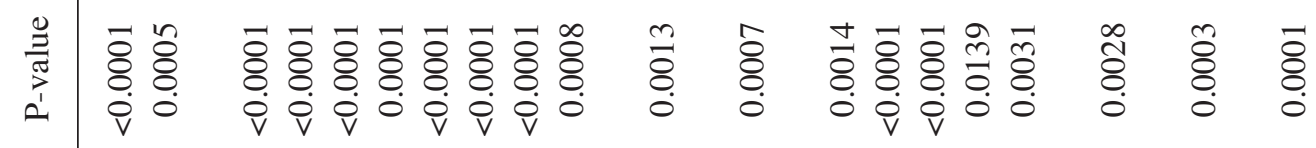

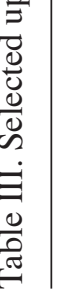

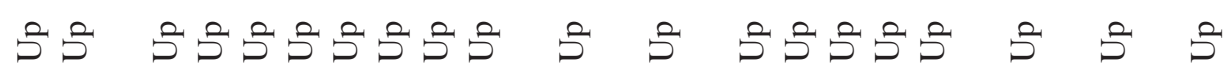

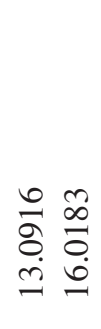




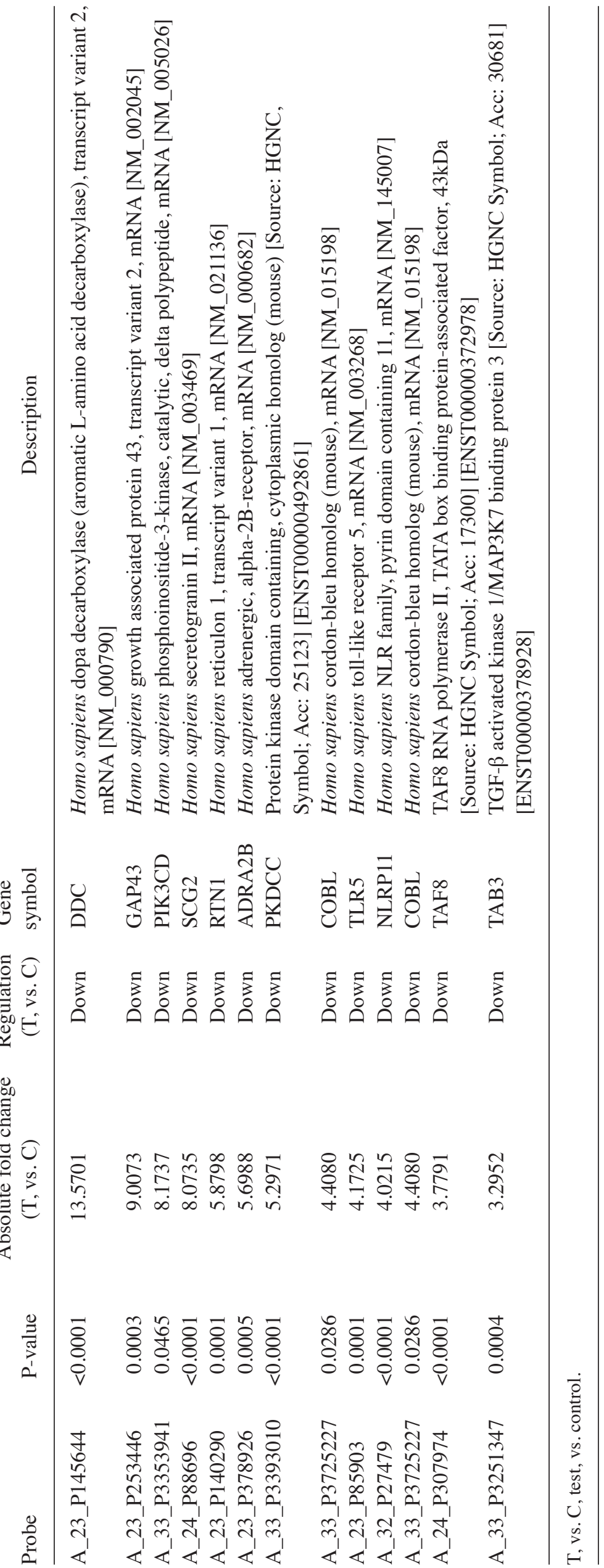


were involved in classic signaling pathways $(\mathrm{P}<0.001$; absolute fold changes $>2$-fold. Certain differentially expressed genes exhibiting upregulated or downregulated expression levels are presented in Tables III and IV.

\section{Discussion}

HSCR occurs when gut neural crest cells are disturbed or interrupted during migration, proliferation, differentiation, survival, colonization and/or apoptosis. Lesions occur predominantly in the distal colon and are characterized by a lack of ganglion cells in the lesion gut. As a non-Mendelian genetic disease, the mode of inheritance of HSCR is complex. HSCR has varied exon expression and exhibits high phenotypic variability with incomplete penetrance. Its clinical symptoms are predominantly manifested as meconium discharge delay (>24 h), abdominal distension, vomiting, malnutrition and developmental disorders (5). Extensive studies (6-8) have identified several key genes that regulate the development of neural crest cells in HSCR pathogenesis, including RET, glial cell line-derived neurotrophic factor (GDNF), GDNF family receptor $\alpha 1$ (GFR $\alpha 1)$, neurturin, endothelin receptor type $\mathrm{B}$, endothelin 3, ZFHX1B, paired-like homeobox 2B, sex determining region Y-box 10 and sonic hedgehog $(\mathrm{SHH})$. However, these genes mutate in only $50 \%$ of known HSCR cases $(3,9)$. The mechanism and association between the various genes and signaling pathways during HSCR formation remain to be fully elucidated, and the identification of further gene mutations and gene mutation combinations may assist in clarifying the etiology and pathogenesis of HSCR. Certain cases of HSCR with the same phenotype are associated with multiple gene mutations, and these characteristics indicate that genetic modification or environmental factors are involved in HSCR formation (3,7-9). Therefore, several aspects of this disease warrant further investigation.

With the emergence of novel genetic models and detection tools for HSCR, novel pathogenetic mechanisms may be suggested. In a previous study, time-lapse image analyses in an established a mouse model demonstrated that the enteric neural crest cells (ENCCS) reach the hindgut through mesenteric transmembrane migration between the midgut and hindgut, rather than a continuous migration process from the midgut to the cecum and then to the hindgut (10). Dysfunctions or mutations occurring during transmesenteric ENCC and circumflex ENCC migration may lead to HSCR formation (10). This novel finding may also affect in vitro experiments aimed at analyzing NCC colonization and ENS formation dynamics.

HSCR can either exist independently or as part of other complex malformation syndromes. Up to $30 \%$ of HSCR patients present with additional abnormalities $(11,12)$, including palate defects (13), congenital heart defects (14), gastrointestinal abnormalities, central nervous system abnormalities, urinary and reproductive system problems, craniofacial deformities and spina bifida (3,14-17). In addition, 2-15\% of HSCR cases are associated with Down's syndrome $(17,18)$. HSCR is part of a group of complex diseases affected by multiple factors and involving multiple genes, including key genes, major gene of the associated genes and minor genes of the associated genes. In the past, molecular biology experiments based on electrophoresis and polymerase chain reaction were only able to analyze the expression or mutation of one or a few genes in each experiment, and a large-scale, high-throughput investigation of functional genes was not achievable. Therefore, large-scale and efficient screening for all possible genes associated with HSCR is required in order to identify the genes associated with human HSCR. The methods of gene expression, sequencing, mutation and polymorphism analyses based on electrophoresis cannot meet the above requirements, therefore, the present study used gene chip technology. In addition to identifying the genes responsible for HSCR and their association in the model, an investigation with continuity is required to identify the corresponding human gene mutations and regulatory network mechanisms. Thus, using whole exome and whole genome sequencing of patient specimens is ideal for screening and identifying the mutations in these genes.

In the present study, the gene expression profiling microarray chip was prepared with several target genes or gene fragments, which were applied on glass using a microarray in an orderly manner and at high densities. The samples to be assessed were labeled with a fluorescent dye to prepare a probe and microarray hybridization, and the hybridization signal was detected using a laser scanner. Hybridization data similar to that of conventional dot blot hybridization were obtained through the computer analysis of the experimental results. Comparing the differences in the target gene expression profiles between groups achieves rapid, efficient, high-throughput and parallel biological information analysis $(19,20)$. With this technique, comparative analysis can be performed for tissue and cell gene expression in individuals, tissues samples, cell cycles, developmental stages, differentiation stages and physiological states under various stimulatory conditions $(19,21)$. This allows identification of changing gene cluster characteristics and patterns in individuals, tissues, stages of development and differentiation, diseases and stimuli, which further clarifies synergy, cross-inhibition and mutual causality $(19,21)$. Therefore, the mechanism underlying disease pathogenesis may be explained at the genetic level. Microarray is currently the most widely used gene chip method, and is used for genetic assessment and functional analysis, including screening for candidate genes of certain diseases (22). Currently, microarray has been widely used in investigations of congenital malformations, including congenital heart disease (23).

HSCR results from ENCC migration and colonization disorders during embryonic gut development. Several studies have demonstrated that HSCR occurrence is associated with various genes, particularly those associated with cell migration and development, including GDNF, the transmembrane receptor tyrosine kinase, RET, and GFR $\alpha 1$, with the signaling pathway formed by these three factors being expressed in $50 \%$ of familial HSCR cases and $30 \%$ of sporadic HSCR cases $(3,20,24)$. Mice missing any one of these factors were found to succumb to morality within $24 \mathrm{~h}$ of birth and lacked gut neurons throughout the entire bowel, between the end of the gastrointestinal tract and the stomach (25). Mice with mutations in endothelin and/or its receptor lack gut neurons in the distal colon, and exhibit neural crest cell migration delays in the gut $(26,27)$ and albinism $(28,29)$. Bone morphogenetic proteins (BMPs) are also involved in regulating ENCC migration and ganglia formation in the enteric nervous system. Specific enteric BMP activity inhibition affects GDNF signals and causes delays 
in ENCC migration, indicating that the interaction between BMP and GDNF is required for correct and complete formation of the enteric nervous system $(30,31)$. The $\mathrm{SHH}$ gene is important in regulating enteric ENCC migration and nerve plexus formation $(32,33)$. Contrary to the effects of GDNF on ENCC migration, the migration is reduced predominantly by regulating the equilibrium between enteric NCC proliferation and differentiation, and the equilibrium between GDNF and SHH determines correct plexus colonization in the enteric nervous system $(32,33)$. CXCR4 is a receptor for the cell surface chemokine pre-B cell growth-stimulating factor/stromal cell-derived factor, which is expressed in endothelial cells and has an indirect impact on ENCC colonization in the hindgut by regulating vascular system development (10). Our previous study demonstrated that mutations in these genes had important implications for HSCR formation (34). The microarray results also demonstrated changes in the expression levels of these associated genes. However, the restriction and balances between these gene and signaling pathways, and their association with HSCR formation requires further investigation.

The present study demonstrated that HSCR occurrence involves mutations in multiple genes. According to the heat map and hierarchical clustering, comparative analysis of the differences in the concurrent gene expression profiles between the control group and HSCR patients on the same chip exhibited parallelism. The investigation of differentially expressed human HSCR genes using microarray analysis allows simultaneous quantification of gene expression levels, as well as the identification of genes with differential expression. Following initial screening for important upstream genes [such as SHH (35), BMP, WNT, NOTCH (36), and RET], the primary and secondary associated genes [such as RET, CXCR4, CR, GDNF, enolase 2 (gamma, neuronal), CAD, B cell lymphoma 2, and glial fibrillary acidic protein] in the associated signaling pathway were gradually screened, according to the association between the gene and various signaling pathways. The present study focussed on the WNT signaling pathway. WNT5A is a member of The WNT gene family 3 (37), and has been implicated in oncogenesis and in several developmental processes, including regulation of cell fate and patterning during embryogenesis (38). WNT5A and its ligand frizzled-5 (hFz5) compose the WNT5A/hFz5 signaling pathway, which is important for organ axis formation. Finally, several analyses were performed to construct a network diagram of HSCR pathogenesis. Therefore, to integrate the separated and scattered HSCR genes and their interactions into a coherent network diagram, genome sequencing remains the most economic option, whereas microarray technology is an efficient and rapid screening method.

\section{Acknowledgements}

The present study was supported by a grant from the National Natural Science Foundation of China (grant no. 81370474).

\section{References}

1. Theveneau E, Mayor R, Neural crest delamination and migration: from epithelium-to-mesenchyme transition to collective cell migration. Dev Biol. 2012 Jun 1; 366(1)34-54.

2. Goldberg EL: An epidemiological study of Hirschsprung's disease. Int J Epidemiol 13: 479-485, 1984.
3. Parisi MA and Kapur RP: Genetics of Hirschsprung disease. Curr Opin Pediatr 12: 610-617, 2000.

4. Jacqueline K. White, Anna-Karin Gerdin, Natasha A. Karp et al. Genome-wide Generation and Systematic Phenotyping of Knockout Mice Reveals New Roles for Many Genes. Cell. 2013 July 18; 154(2): 452-464

5. Washita T, Kruger GM, P ardal R, Kiel MJ, Morrison SJ. Hirsch-sprung disease is linked to defecta in neural crest cell function. Science.2003;301(5635)972-976.

6. Erin Mundt, Michael D, Bate. Genetics of Hirschsprung disease and anorectal malformations. Seminars in Pediatric Surgery(2010)19, 107-117.

7. Angrist M, Bolk S, Halushka M, Lapchak PA and Chakravarti A: Germline mutations in glial cell line-derived neurotrophic factor (GDNF) and RET in a Hirschsprung disease patient. Nat Genet 14: 341-344, 1996.

8. Salomon R, Attié T, Pelet A, Bidaud C, Eng C, Amiel J, Sarnacki S, Goulet O, Ricour C, Nihoul-Fékété C, et al: Germline mutations of the RET ligand GDNF are not sufficient to cause Hirschsprung disease. Nat Genet 14: 345-347, 1996.

9. Bolk S, Pelet A, Hofstra RM, Angrist M, Salomon R, Croaker D, Buys $\mathrm{CH}$, Lyonnet $\mathrm{S}$ and Chakravarti A: A human model for multigenic inheritance: Phenotypic expression in Hirschsprung disease requires both the RET gene and a new 9q31 locus. Proc Natl Acad Sci USA 97: 268-273, 2000.

10. Nishiyama C, Uesaka T, Manabe T, Yonekura Y, Nagasawa T, Newgreen DF, Young HM and Enomoto H: Trans-mesenteric neural crest cells are the principal source of the colonic enteric nervous system. Nat Neurosci 15: 1211-1218, 2012.

11. Passarge E: The genetics of Hirschsprung's disease. Evidence for heterogeneous etiology and a study of sixty-three families. N Engl J Med 276: 138-143, 1967.

12. Spouge D and Baird PA: Hirschsprung disease in a large birth cohort. Teratology 32: 171-177, 1985.

13. Kerstjens-Frederikse WS, Hofstra RM, van Essen AJ, Meijers JH and Buys CH: A Hirschsprung disease locus at 22q11? J Med Genet 36: 221-224, 1999.

14. Ryan ET, Ecker JL, Christakis NA and Folkman J: Hirschsprung's disease: Associated abnormalities and demography. J Pediatr Surg 27: 76-81, 1992.

15. Badner JA, Sieber WK, Garver KL and Chakravarti A: A genetic study of Hirschsprung disease. Am J Hum Genet 46: 568-580, 1990.

16. Sarioglu A, Tanyel FC, Büyükpamukçu N and Hiçsönmez A: Clinical risk factors of Hirschsprung-associated enterocolitis. I: Preoperative enterocolitis. Turk J Pediatr 39: 81-89, 1997.

17. Amiel $\mathrm{J}$ and Lyonnet S: Hirschsprung disease, associated syndromes, and genetics: A review. J Med Genet 38: 729-739, 2001.

18. Fu M, Lui VC, Sham MH, Cheung AN and Tam PK: HOXB5 expression is spatially and temporarily regulated in human embryonic gut during neural crest cell colonization and differentiation of enteric neuroblasts. Dev Dyn 228: 1-10, 2003.

19. Schena M, Shalon D, Davis RW and Brown PO: Quantitative monitoring of gene expression patterns with a complementary DNA microarray. Science 270: 467-470, 1995.

20. Heller RA, Schena M, Chai A, Shalon D, Bedilion T, Gilmore J, Woolley DE and Davis RW: Discovery and analysis of inflammatory disease-related genes using cDNA microarrays. Proc Natl Acad Sci USA 94: 2150-2155, 1997.

21. De Risi J, Penland L, Brave PO, et al. Use of a cDNA microarray to analyse gene expression patterns in human cancer[ J] $\mathrm{DNat}$ Genet, 1996, 14 (4) : 457-460.

22. Wu TD: Analysing gene expression data from DNA microarrays to identify candidate genes. J Pathol 195: 53-65, 2001.

23. Miertus $\mathbf{J}$ and Amoroso A: Microarray-based genetics of cardiac malformations. Ital Heart J 2: 565-567, 2001.

24. Bassett DE Jr, EisenM $\square$ Boguski MS. Gene expression informatics it's all in your mind[ J] $\square$ Nat Genet, 1999, 21(Suppl 1):51-55.

25. Tomac AC, Grinberg A, Huang SP, Nosrat C, Wang Y, Borlongan C, Lin SZ, Chiang YH, Olson L, Westphal H and Hoffer BJ: Glial cell line-derived neurotrophic factor receptor alphal availability regulates glial cell line-derived neurotrophic factor signaling: Evidence from mice carrying one or two mutated alleles. Neuroscience 95: 1011-1023, 2000.

26. Barlow A, de Graaff E and Pachnis V: Enteric nervous system progenitors are coordinately controlled by the $\mathrm{G}$ protein-coupled receptor EDNRB and the receptor tyrosine kinase RET. Neuron 40: 905-916, 2003. 
27. Druckenbrod NR and Epstein ML: Age-dependent changes in the gut environment restrict the invasion of the hindgut by enteric neural progenitors. Development 136: 3195-3203, 2009.

28. Lee HO, Levorse JM and Shin MK: The endothelin receptor-B is required for the migration of neural crest-derived melanocyte and enteric neuron precursors. Dev Biol 259: 162-175, 2003.

29. Ro S, Hwang SJ, Muto M, Jewett WK and Spencer NJ: Anatomic modifications in the enteric nervous system of piebald mice and physiological consequences to colonic motor activity. Am J Physiol Gastrointest Liver Physiol 290: G710-G718, 2006.

30. Goldstein AM, Brewer KC, Doyle AM, Nagy N and Roberts DJ: BMP signaling is necessary for neural crest cell migration and ganglion formation in the enteric nervous system. Mech Dev 122: 821-833, 2005

31. Faure C, Chalazonitis A, Rhéaume C, Bouchard G, Sampathkumar SG, Yarema KJ and Gershon MD: Gangliogenesis in the enteric nervous system: Roles of the polysialylation of the neural cell adhesion molecule and its regulation by bone morphogenetic protein-4. Dev Dyn 236: 44-59, 2007.

32. Ramalho-Santos M, Melton DA and McMahon AP: Hedgehog signals regulate multiple aspects of gastrointestinal development. Development 127: 2763-2772, 2000.
33. Fu M,Lui VC, Sham MH,Pachnis V and Tam PK: Sonic hedgehog regulates the proliferation, differentiation and migration of enteric neural crest cells in gut. J Cell Biol 166: 673-684, 2004.

34. Ding X, Zhao Z, Duan W, Wang S, Jin X, Xiang L and Jin X: Expression patterns of CXCR4 in different colon tissue segments of patients with Hirschsprung's disease. Exp Mol Pathol 95: 111-116, 2013.

35. Choudhry Z, Rikani AA, Choudhry AM, Tariq S, Zakaria F, Asghar MW, Sarfraz MK, Haider K, Shafiq AA and Mobassarah NJ: Sonic hedgehog signalling pathway: A complex network. Ann Neurosci 21: 28-31, 2014.

36. Ngan ES, Garcia-Barceló MM, Yip BH, Poon HC, Lau ST, Kwok CK, Sat E, Sham MH, Wong KK and Wainwright BJ: Hedgehog/Notch-induced premature gliogenesis represents a new disease mechanism for Hirschsprung disease in mice and humans. J Clin Invest 121: 3467-3478, 2011.

37. Clark CC, Cohen I, Eichstetter I, Cannizzaro LA, McPherson JD, Wasmuth JJ and Iozzo RV: Molecular cloning of the human proto-oncogene Wnt-5A and mapping of the gene (WNT5A) to chromosome 3p14-p21. Genomics 18: 249-260, 1993.

38. Bhatt PM and Malgor R: Wnt5a: A player in the pathogenesis of atherosclerosis and other inflammatory disorders. Atherosclerosis 237: 155-162, 2014. 